A Magnetized Umbrella.

Some sensational paragraphs have recently appeared in the press about a fortunate umbrella, whose proclivities, being " magnetic," were detrimental to the decent and correct bearing of the compass or "binnacle" on board a Scotch passenger steamer, the Princess Beatrice, plying from Larne to Stranraer. We mention "fortunate," as the umbrella in question has been purchased from the passengerowning it and forwarded by the captain of the sbip to Sir William Thomson, F.R.S., in whose laboratory at Glasgow it will doubtless undergo those pleasing sensations naturally incident to a thorough test and report on its scientific complaint.

The helmsman, on a certain voyage of the above named steamer, noticed that the compass by which he was steering was invariably affected by the near approach of a certain passenger, and finally the "fault" was located in the aforesaid passenger's umbrella,
which on subsequent trials was found to be strongly which on subsequent trials was found to be strongly magnetic. As umbrellas are usually made of hard steel, this result is not to be wondered at; but even if not distinctly magnetic, the amount of steel in the umbrella would, on near approach to the compass, certainly affect it. Presuming that that umbrella had at a recent date made the acquaintance of an active dynamo, its normal condition would have been altered into a "magnetic" one, and such a state of things we have witnessed ourselve

It is well known that the too near approach of iron to magnetic needles affects them more or less, and we call to mind an amusing incident related by Captain Marryat, where a native servant placing a frying pan under the binnacle disturbs the superposed magnetic needle. We have seen the magnet of a Thomson galvanometer seriously affected by the pins in a lady's hair, and have ourselves had to replace steel spectacles by non-metallic ones. Indeed, in ordinary testing with a similar galvanometer, it is usual to remove knives, keys, etc., from one's person before making any tests with so delicate an instrument. It was but recently that the vagaries of a not oversensitive horizontal galvanometer fairly for a time puzzled us; the removal of everything in the shape of iron and steel did not alter the erratic behavior of the needle, our near approach for the purpose of examining the deflection produced the same erratic results, until it seemed that it was almost like personal magnetism; but finally the culprit was found in a round felt hat that we were wearing, but as this was supposed to be all "felt," it at the-least seemed strange, although the fause was found in a fine steel ring in the outer edge of the hat to give the rim shape, which was strongly "magnetic," hav give the rim shape, which was strongly "magnetic," hav ing undoubtedly become so from frequent intercourse with active and powerful dynamos. Instances of these kinds are extremely numerous, and it points to a conclusion that as an aid to correct navigation, it would not be advisable to place an umbrella stand round the "binnacle," or that box which contains the steerin compass of a ship.

As a practical result of the behavior of this umbrell we shall probably hear of the passengers to and from the Isle of Man and other places endeavoring to discover whether they are also blessed with a magnetic umbrella. In addition to the usual instruction, "not to speak to the man at the wheel," we shall not be surprised to see the notice, "Passengers are requested not to poke fun at the compass with their umbrellas." Mechanical World.

\section{a Color Blind Fireman.}

Mr. Julius King, who is the examiner for color blindness for the Lake Shore and Michigan Southern Railroad, has discovered a remarkable case. The patient is an employe of the railroad company. $\mathrm{He}$ King made three tests in his case. First, colored glass King made three tests in his case. First, colored glass
globes were placed over a gas jet, and the man, at globes were placed over a gas jet, and the man, at
a distance of 20 feet away, asked to tell the colors. He named the red globe correctly when it was first used, but on second trial declared it to be green. Then railway signal flags of different colors were waved before him. He called the red flag green, the green flag red, and when two flags, both red, but of different shades, were waved, the fireman insisted that they were green. Red and green flags held up together he declared to be green. The next test was made with a small rack in which hung zephyr worsted of different colors. The standard color of green was pointed out to the man, and he was asked to select the worsted in the rack of the same color. He immediately picked out bright red, old gold, and light brown bunches. The unfortunate fireman had to be brown bunches. The unfortunate fireman had to be very large number of men for color blindness, and that very large number of men for color blindness, and that
about four men in every one hundred are defective in about four men in every one hundred are defective in
their eyesight in this respect. But very few people their eyesight in this respect. But very few people
are as color blind as the fireman. He said that women were seldom found color blind, as they constantly trained their eyes in selecting colors in ribbons and dry goods, and in discriminating between delicate shades and tints. In answer to a question, Mr. King explained: "The theory of the cause of color blindness is that parts of the retina of the eye respond each to different colors. When any of these parts are deficient, absent, or undeveloped, the person cannot see the color that it belongs to, leaving som
sponsive part to act."-Des Moines Leader.

\section{AN IMPROVED WABH-BOTTLE FOR CHEMTCAL}

\section{LABORATORIES.}

. $x$.

By this simple device the washing of precipitates and the cleansing of vessels used in the process of wash-bottle, can now be done with much more facilit and in a shorter time.

It consists essentially of a thin glass flask, C, placed bout three feet above the level of the working desk, nd closed by a three-hole rubber stopper. Through

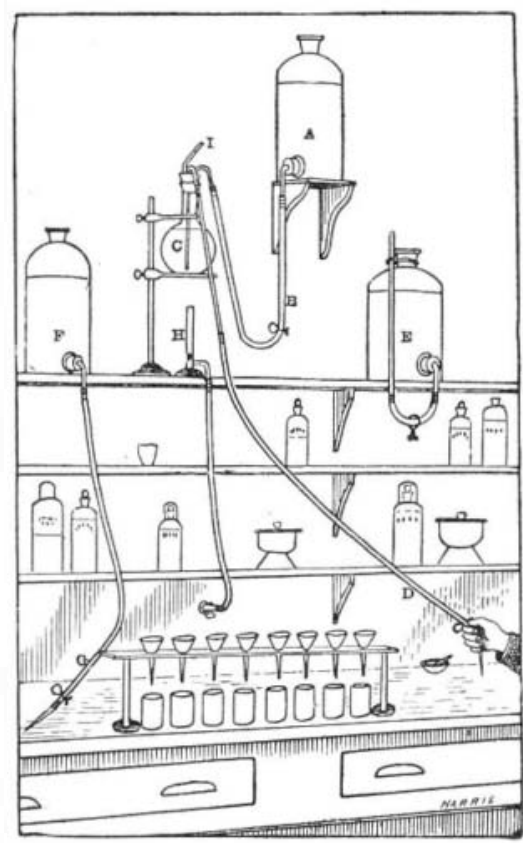
one of the holes issues a rubber tube, D (or Construction, Fourth Lighthouse District tendent of sented a clear mathematical view of the agencies which ber c on nec- influence the tides and the local conditions which must tions), descend- have place in tidal predictions. It is now so generally ing to the desk acknowledged that the tides are caused by the differand ending in ence in the attractions of the sun and moon on those a glass nozzle. parts of the earth nearest to them, and on their antipoConnection is $\begin{aligned} & \text { parts of the earth nearest to them, and on their antipo- } \\ & \text { dest any detail statements concerning the main }\end{aligned}$ made by a sec- theory seem entirely superfluous; since, however, the ond hole in the heavenly conditions causing the tides are by no means stopper with a simple or uniform, but are subject to constant changes, reservoir bot- it becomes a matter of some interest to investigate the tle, A, placed causes of those variations, and to learn their quantitaabove the top tive effect.

of the wash- The first cause which suggests itself is the changes bottle. In the in the distance of sun and moon from the earth. The third hole is moon's orbit is an ellipse, the earth being at one focus, placed a glass and during ber revolution, therefore, she constantly tube bent at an changes her distance from the earth. This cycle of angle to keep change, represented by one lunar revolution, occupies out dust. On a little over twenty-seven days, during which time the filling the flask moon passes from her greatest distance to her smallest from the reser- distance, and back again to her greatest distance. In from the reser-
voir-the flow
addition to this disturbing influence, the axes of the $\begin{array}{ll}\text { voir-the flow } & \text { addition to this disturbing influence, the axes of the } \\ \text { being stopped } & \text { moon's orbit are not constant, owing to solar attrac- }\end{array}$ by a pinch cock-the water is started by suction from tion, but vary in a period of about nine years.

below, and the stream through the nozzle can be reguated or stopped at will by a pinch cock placed conveniently to the hand, the height of the water flask furA Bunsen burner, $\mathrm{H}$, is placed underneath the flask, and the water can be heated when it is so desired. Hot water as well as cold can thus be used in treating pre cipitates. Other solutions cán be employed equally a ell as water. (See bottle F.)

The advantages of this system are:

1st. The saving of much time and consequent labor attending the use of an ordinary wash-bottle, especially where several analyses are carried on at the same time
the exertions required by the mouth and lungs bein thereby avoided.

2d. No air exists in the tube, as in an ordinary washbottle, and consequently the full force of the liquid is utilized immediately

$3 d$. When used with a wash solution of ammonia water, no trouble is experienced with free ammonia The large bottle, E, with the accompanying tube shows a convenient arrangement for holding any solu tion and delivering the same.

\section{A CONVENIENT ARTICLE FOR HOSPITALS AND SIC} ROOMS.

The illustration herewith shows an improved form of cover for vessels for use in chambers and sick rooms,

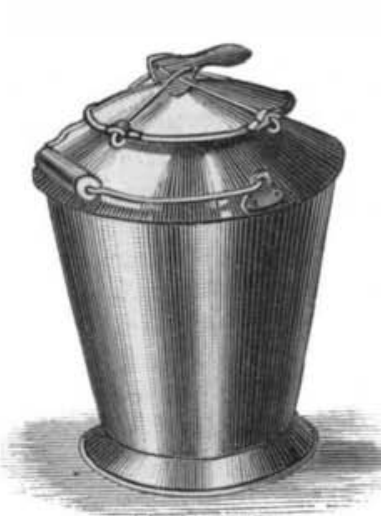

the device embracing an easily operated lever attachment, on the top of the cover, in connection with the handle, whereby the cover is made to fit closely on a rubber to corfine all the berv odors within the vessel. Thu device is an exThe device is an ex expersive to make, and expersive to make, and the weight of the cover and is so made that the latter can at any tim

This invention has been patented by Mr. Agur Judson, of 111 Commerce Street, Newark, N. J. Weople, says a sensible writer, the want of down struck head first, made a complete soriersault well defined system or method is one of the chief and threw himself on the ground flat on his back; the causes of their getting behindhand with their work. rider, being pitched ahead, cleared the horse. Fortry, will atic method of working, combined with indus- tunately neither rider nor animal was seriously injured. finish it with ease; but without system and application, and endeavored to make the horse try the same jump the worker may be in a continual rush, and yet accom- again, but the creature showed more sense than the plish little.
man by resolutely declining the dangerous job. 